\title{
La Formación Didáctico-Matemática de Docentes: resultados teóricos
}

\author{
Nancy Montes de Oca Recio \\ nancymontesde@gmail.com \\ https://orcid.org/0000-0002-5651-3927 \\ Universidad de Camagüey Ignacio Agramonte Loynaz (UCIAL) \\ Camaguey, Cuba
}

Recibido: 26/04/2020 Aceptado: 20/05/2020

\begin{abstract}
Resumen
El objetivo del presente artículo es comunicar el proceso y los resultados teóricos de una investigación relacionada con la formación didáctico matemática de docentes, generada a partir de las insuficiencias detectadas en el desempeño de docentes de Matemática en el ejercicio de la profesión. La investigación aborda aspectos relacionados con: la comprensión de los objetos de la Geometría Plana; la gestión didáctica de las demostraciones matemáticas, la promoción de una valoración positiva hacia la Matemática, la producción de problemas, el dominio de conceptos básicos del Álgebra Lineal y la expresión al comunicarse en matemática, desde una perspectiva teórica que integra el enfoque histórico cultural del desarrollo humano y los enfoques ontosemiótico, comunicativo y contextualizado de la enseñanza-aprendizaje de la Matemática. Los resultados responden a la labor realizada por un equipo de trabajo constituido por profesores que imparten docencia en carreras de formación de docentes de Matemática, maestrías y doctorados de la Universidad de Camagüey y la Universidad Autónoma de Santo Domingo.
\end{abstract}

Palabras clave: Formación Didáctico-Matemática, Educación Matemática, Docentes de Matemática, Gestión Didáctica

\section{A Formação Didático-Matemática de Pedagogos: implicações teóricas}

\begin{abstract}
O objetivo deste artigo presente é comunicar o processo e os resultados teóricos de uma investigação relacionados com o processo de aprendizagem didático-matemático de pedagogos; este estudo começou a levar em conta as insuficiências descobriu no processo de ensinoaprendizagem de Matemática administrado por professores escolares. Esta investigação chega aspectos relacionados com: a compreensão de objetos de Geometria Planos; a administração didática de demonstrações de Matemáticas, a promoção de uma estimação positiva para Matemática, a geração de problemas didáticos, o dominando de conceitos básicos de Álgebra Linear e comunicação matemática, de uma perspectiva teórica que integra a aproximação histórica cultural de desenvolvimento humano e o ontosemiotico, comunicativo e contextual focaliza do ensino-aprendizagem de Matemática. Os resultados apresentados são um resultado do trabalho levado a cabo por um time constituiu por professores que ensinam Matemática em pre-serviço, mestres e cursos de doutorado em Universidade de Camaguey e a Universidade Autônoma de Santo Domingo.

Palavras chave: Formação Didático-Matemática, Educação Matemática, Educação de Matemática, Administração didática.
\end{abstract}




\title{
The Didactic and Mathematical Formation of Teachers: theoric results
}

\begin{abstract}
The objective of this present article is to communicate the process and the theoretical results of an investigation related with the didactic-mathematical learning process of educators; this study began taking into account the inadequacies detected in the teaching-learning process of Mathematics managed by school teachers. This investigation approaches aspects related with: the understanding of Plane Geometry objects; the didactic management of Maths demonstrations, the promotion of a positive valuation towards Mathematics, the generation of didactic problems, the mastering of basic concepts of Lineal Algebra and mathematical communication, from a theoretical perspective that integrates the cultural historical approach of human development and the ontosemiotic, communicative and contextual focuses of the teaching-learning of Mathematics. The results presented is a result of the work carried out by a team constituted by professors that teach Mathematics in pre-service, masters and doctorates courses at Camaguey University and the Autonomous University of Santo Domingo.
\end{abstract}

Key words: Didactic-Mathematical Formation, Mathematical Education, Education of Mathematics, Didactic Management.

\section{Introducción}

En la actualidad los desempeños de los docentes de Matemática son cuestionados en muchos casos, por no responder adecuadamente a las necesidades de aprendizaje de sus estudiantes y a la formación integral en su sentido más amplio.

Lo expresado es avalado por diversas investigaciones (Font y Godino, 2006; Godino, 2009, 2013; Font, 2011) que han corroborado, desde la práctica, las insuficiencias que presentan los docentes de Matemática en la gestión de la clase, en la utilización de métodos y estrategias eficaces, ajustados a las exigencias de una Didáctica de la Matemática centrada en las características específicas del aprendizaje matemático, de sus objetos y métodos particulares; así como de las particularidades de los docentes. Al respecto expresan la necesidad de un tipo de formación que integre lo didáctico y lo disciplinar (matemático).

Ello ha conllevado que en estos últimos años se trabaje tenazmente para que estos trasciendan en su actuación mediante una gestión didáctica novedosa, que propicie un aprendizaje formativo, para proponerse logros en el valor y sentido de lo que se aprende y la forma en que se aprende; que implique poner al estudiante en contacto con la realidad que lo rodea, para que desarrolle habilidades, valores y actitudes mediante tareas y proyectos que lo motiven a la investigación y a la búsqueda de conocimientos de una manera crítica y reflexiva (Montes de Oca y Machado, 2014). 
Es reconocido que diversas instituciones ofrecen a los docentes de esta área programas de capacitación académica, pero no siempre a la medida de esas necesidades, ni fundamentadas científicamente a partir de modelos que develen una lógica integradora de lo matemático y lo didáctico; de ahí que, desafortunadamente, no se lleve a cabo un verdadero mejoramiento de la práctica cotidiana.

Los seminarios, talleres, cursos o diplomados proporcionan de alguna manera una preparación sobre las nuevas tendencias curriculares, metodológicas o pedagógicas que se implantan paulatinamente, tanto en el nivel internacional como nacional; sin embargo, esa preparación sigue siendo, en ocasiones, fragmentada y carente de una perspectiva clara del hecho formativo contextualizado, lo que la hace no siempre relevante y pertinente.

Además, los aspectos a trabajar se quedan, en muchos casos, en lo general y no se observa un proceso formativo sistémico, sistemático y ajustado a las particularidades de la Didáctica de la Matemática en la actualidad.

Desde una perspectiva similar Moura (2011, p, 57) plantea que “...el saber específico de matemática encierra un saber pedagógico..." y puntualiza en la necesidad de una formación didáctico-matemática, la cual no se lleva a cabo espontáneamente, ni solo a través de cursos de didáctica que en la mayoría de los casos no ejercen la influencia necesaria en la actuación de los docentes.

Aunque existe consenso al considerar que la formación didáctico-matemática de los docentes requiere contemplar diversos contenidos didácticos y matemáticos que están estrechamente relacionados entre sí, la selección y organización de los mismos es una problemática aún no resuelta en las Ciencias Pedagógicas por la dificultad epistemológica que posee el discriminar y relacionar los saberes generales con los específicos.

En ese sentido, como parte del proyecto "Perfeccionamiento de la enseñanza de la Matemática” del Programa Nacional “Problemas Actuales del Sistema Educativo Cubano“ y con la participación de un grupo de docentes investigadores de la Universidad de Camagüey, Cuba y de la Universidad Autónoma de Santo Domingo de la República Dominicana se desarrolló a partir del año 2014 una investigación para dar respuesta al problema relacionado con las insuficiencias en el desempeño de estudiantes y docentes en el ejercicio de la profesión desde una perspectiva didáctico-matemática. 
En atención a esas carencias, el objetivo de la investigación estuvo encaminado a contribuir a mejorar el desempeño de docentes en ejercicio y de los estudiantes de las carreras de Educación Matemática, mediante la implementación de estrategias, entrenamientos y metodologías sustentadas en modelos teóricos novedosos que integren lo didáctico y lo disciplinar (matemático).

En este artículo se presenta el marco de referencia que dio sustento a los referidos modelos, derivado del proceso investigativo, a partir de la sistematización teórica realizada y que articula la perspectiva histórico-cultural del desarrollo humano, con los enfoques ontosemiótico del conocimiento y la instrucción matemática (EOS), comunicativo y contextualizado de la enseñanza-aprendizaje de la Matemática.

Para cumplimentar el objetivo y alcanzar los resultados científicos fueron utilizados métodos del nivel teórico y empíricos, tales como: la revisión documental, el análisis del producto de la actividad, la modelación sistémico-estructural, el hipotético-deductivo, el análisis-síntesis, la abstracción-concreción y la inducción-deducción.

Para desarrollar la investigación se siguió una metodología de trabajo que contó con las fases que se detallan a continuación:

\section{Fase 1: Determinación de un marco de referencia para la formación didáctico-matemática de los docentes.}

Esta fase estuvo orientada a precisar el marco de referencia y el cuerpo categorial de la investigación desde el contexto específico en que se desarrolla. A continuación, se expone una síntesis de los principales resultados y aportes obtenidos en esta fase de la investigación:

Los fundamentos se expresan; a través de la articulación de diferentes propuestas teóricas sustentadas desde lo más general en el enfoque histórico cultural del desarrollo humano (Vigotsky, 1987) el enfoque ontosemiótico del conocimiento y la instrucción matemática (Godino, 2009), el enfoque comunicativo (Roméu, 2011; González 2010, Rincón, 2018) y el contextualizado (Camarena, 2009) de la enseñanza-aprendizaje de la Matemática.

De Vigotsky (1987), se asume especialmente lo relacionado con la importancia del medio social en el desarrollo humano, la unidad de lo cognitivo y lo afectivo y la importancia de la actividad mediada, donde en interacción con su contexto sociocultural el sujeto internaliza las funciones psicológicas superiores a través del intercambio de significados y experiencias con 
la participación crítica y activa en espacios comunicativos, concibiéndose el aprendizaje de la Matemática desde una perspectiva social, a partir de las interacciones entre los sujetos implicados, donde los objetos y procesos matemáticos se pueden considerar como entes que emergen progresivamente de sistemas de prácticas socialmente compartidas en la clase.

Además, se considera la importancia del lenguaje matemático como una herramienta para la comunicación, el cual se enseña y se utiliza en diferentes contextos, donde los procesos de comprensión y argumentación juegan un papel fundamental. Se priorizan los procesos de análisis y comprensión mediante la realización de situaciones didáctico-matemáticas teniendo en cuenta el contexto donde se producen y una intención comunicativa.

A la vez se tiene en cuenta a la Matemática como herramienta y disciplina formativa, desde la que se considera que la contextualización se expresa en la integración, interacción, relaciones y nexos de los contenidos con los procesos y fenómenos de la vida cotidiana o con los problemas de la actividad profesional expresados en el contenido de las asignaturas de la profesión.

Desde esos presupuestos y desde los estudios de Byas y Blanco (2017) se asumen los elementos que caracterizan la ontología y epistemología de la Matemática, que son fundamentales para la formación didáctico-matemática de los docentes por ser necesarios para gestionar el proceso de enseñanza-aprendizaje y que a continuación se enuncian:

- Carácter no ostensivo de los objetos matemáticos.

- Carácter relativo de la verdad matemática.

- La matemática medio y objeto en sí misma.

- Posee un lenguaje propio.

- Tiene una estructura sistémica y una lógica de desarrollo intrínseca.

Se definen o redefinen conceptos esenciales, que se constituyen en las categorías principales del marco declarado, entre los que se destacan:

Formación didáctico-matemática de los docentes como el "̈proceso y resultado con un carácter transformador, orientado a mejorar el desempeño de los docentes a través de una gestión didáctica que tenga en cuenta el manejo de situaciones y criterios de idoneidad en correspondencia con las exigencias del contexto y los elementos que caracterizan las tendencias actuales de la Didáctica de la Matemática". 
La gestión didáctica como el "proceso de orientación, planeación, organización y ejecución, donde el control y la valoración se conciben transversalmente; se concreta en un contenido y se desarrolla a través del sistema de relaciones e interacciones que se establecen entre estudiantes, estudiantes y docentes, entre docentes y otras fuentes humanas o tecnológicas, con un carácter dinámico que privilegia la comunicación para alcanzar los objetivos de aprendizaje.".

El contenido matemático en sentido amplio, que incluye, “los objetos matemáticos (conceptos, términos, expresiones, relaciones, propiedades, algoritmos), procesos generales (comprensión, argumentación, generalización, sistematización, gestión) y los propios de la matemática que se articulan o integran con los generales (resolución de problemas matemáticos, modelación matemática, la argumentación matemática, entre otros). .

Las situaciones didáctico-matemáticas como "formas de presentación del contenido y un recurso metodológico que coadyuva a una participación activa, reflexiva, responsable y crítica de los estudiantes y docentes, pues posibilitan prestar atención a los requerimientos que constituyen el núcleo fundamental de cada uno de los campos de acción del docente en el tratamiento didáctico de los objetos y procesos matemáticos, con especial atención a los relacionados con la comunicación que se lleva a cabo en el contexto del aula, el trabajo con los diferentes registros de representación semiótica y la utilización del lenguaje matemático, entre otros.

Como resultado del estudio realizado se declaran como características fundamentales de las situaciones didáctico-matemáticas, en el marco de la presente investigación las siguientes:

- Permiten organizar el tratamiento didáctico de los objetos y procesos matemáticos en atención a los diferentes contextos.

- Otorgan prioridad a los procesos cognitivos-comunicativos y socioculturales en los que el lenguaje matemático participa.

- Permiten la articulación entre los diferentes registros de representación de los objetos matemáticos y la transferencia del conocimiento utilizado en un contexto a otro contexto diferente.

- En ellas se concreta la orientación comunicativa y contextualizada de los objetivos, contenidos y métodos.

- Permiten valorar las interacciones de los docentes, los alumnos y de estos con los recursos didácticos.

- Propician la gestión del conocimiento a través de múltiples fuentes y en especial a partir del empleo de las tecnologías de la información y las comunicaciones. 
- Se fundamentan los desempeños idóneos del docente universitario como expresión de las competencias que deben desarrollar en el escenario actual a partir de la propuesta de Machado y Montes de Oca (2016), entre los que se destacan:

- Gestionar el proceso de enseñanza-aprendizaje utilizando métodos y procedimientos del aprender a aprender y aprender a enseñar promoviendo el aprendizaje colaborativo, cooperativo, significativo y desarrollador.

- Aplicar las herramientas técnicas y tecnológicas de punta en el proceso formativo y la investigación, adoptando una posición abierta para su incorporación en todos los planos de su labor profesional.

- Dirigir su labor hacia la mejora contextual, con compromiso social y retribuyendo con responsabilidad a los beneficios de su formación en el entorno social.

Otro constructo de vital importancia fue el de "idoneidad didáctica", utilizado como un medio o guía para la valoración, ya que posee un dimensionamiento teórico, criterios y una operacionalización que se encuentra argumentada en diversas investigaciones (Godino, 2009; 2013; Godino, Bencomo, Font y Wilhelmi, 2007; Font, 2011). Ella, según sus autores, es una herramienta que permite establecer criterios valorativos de la gestión didáctica de la actividad matemática de los docentes desde una perspectiva consensual, posibilitando el paso a una didáctica que se orienta sistémicamente hacia la intervención efectiva en el aula desde las dimensiones epistémica, ecológica, cognitiva, afectiva, interaccional y mediacional, insertas en los procesos de estudio de la Matemática.

En el presente estudio, luego de un proceso de observación, análisis y síntesis se agruparon en dos las dimensiones y se determinaron indicadores a partir de los criterios establecidos por Pino-Fan, Castro, Godino, y Font (2013) y Breda, Font y Pino-Fan (2018) y que a continuación se resumen:

Idoneidad epistémica-ecológica-cognitiva se manifiesta en:

- La utilización de definiciones y procedimientos correctamente enunciados y adaptados al nivel educativo al que se dirigen.

- La adecuación de las explicaciones.

- La adecuada selección y utilización de la secuencia de tareas o situaciones didácticas que potencien la realización de procesos relevantes en la actividad matemática, así como de otros procesos cognitivos y metacognitivos.

- La adecuada selección, implementación y evaluación de los contenidos de acuerdo a las directrices curriculares, así como en la innovación didáctica.

- Idoneidad afectiva-interaccional-mediacional está asociada a: 
- La conducción y comprensión de situaciones didácticas en el aula.

- La capacidad de relacionarse constructivamente con los estudiantes.

- Interactuar de manera cooperada en condiciones de una marcada diversidad, heterogeneidad.

- Manejar las tecnologías de la información las comunicaciones.

- La resolución de conflictos que inciden en el aprendizaje.

- La toma de decisiones en el contexto educativo y del entorno social, relacionadas con la promoción de metodologías colaborativas y la búsqueda de alternativas para superar las dificultades que surgen durante el desarrollo de las actividades docentes con apertura y flexibilidad.

Específicamente para esta dimensión se determinaron criterios para evaluar el desempeño del profesor al comunicarse en matemática (Sobrado, Sarduy y Montes de Oca, 2016) y que a continuación se enuncian:

- Precisión: Atribuir a cada término y símbolo matemático usado, el significado exacto y un sentido determinado. No existe ambigüedad, indeterminación, confusión en cuanto al objeto matemático al que se refiere o la forma de usarlo.

- Argumentación: Dar razones para afirmar o refutar la veracidad de un juicio dado, a partir del cumplimiento de los elementos necesarios y suficientes.

- Jerarquización: Exponer las ideas importantes ordenadamente.

- Concreción: Declarar lo esencial de las ideas.

Teniendo en cuenta las dimensiones y criterios declarados, se realizó, un diagnóstico de la situación actual del desempeño de los docentes en la Universidad Autónoma de Santo Domingo, República Dominicana. Para ello se utilizaron métodos tales como, el análisis del producto de la actividad y la revisión documental (Rincón, 2018; Núñez, 2018; Pérez, 2018; Báez, 2018; Almonte, 2018; Lachapell, 2018).

Los resultados del diagnóstico corroboraron que existen insuficiencias en el desempeño de los docentes, relacionados con la atención a los problemas afectivos matemáticos de los estudiantes, con el tratamiento didáctico de la comprensión de los objetos de la Geometría Plana y de las demostraciones matemáticas; también se encontraron insuficiencias en el dominio de conceptos básicos y producción de problemas del Álgebra Lineal, todo lo cual fundamenta de manera particular y contextuada las insuficiencias declaradas en la introducción y así dar paso a la siguiente fase de la investigación. 


\section{Fase 2: Determinación de los ejes particulares de la formación didáctico-matemática y elaboración de modelos teóricos.}

A partir de la caracterización epistemológica y el diagnóstico realizado, se establecieron como ejes particulares de la formación didáctico-matemática los siguientes:

- El tratamiento didáctico del desarrollo de la comprensión de objetos geométricos.

- La gestión didáctica de las demostraciones matemáticas.

- La formación de la competencia docente promoción de la valoración positiva de la matemática.

- La competencia formación de conceptos básicos del Álgebra Lineal.

- La producción de problemas durante el proceso de enseñanza aprendizaje del Álgebra Lineal.

- La formación integrada (matemático-didáctica) del maestro de Educación Primaria.

Cada uno de estos ejes generó una investigación particular que concluyó con la defensa de seis tesis en opción al Grado Científico de Doctor en Ciencias Pedagógicas (Rincón, 2018; Núñez, 2018; Pérez, 2018; Báez, 2018; Almonte, 2018; Lachapell, 2018). De esta manera, las tesis poseen un núcleo conceptual-metodológico integrador; en este caso la formación didácticomatemática de los docentes, el marco de referencia argumentado constituye la base metodológica de las mismas, pero siempre con la flexibilidad necesaria que permitiría que cada investigador pudiera adaptar las principales categorías a las condiciones específicas del contexto donde se realizaría la intervención y los objetivos particulares que se perseguían.

Las mismas, contribuyen, como síntesis, a la elevación del conocimiento y de la praxis en un plano determinado de desarrollo y cuyos resultados teóricos fundamentales se presentan de forma sucinta a continuación:

Modelo didáctico del desarrollo de la compresión de los objetos de la Geometría Plana (Rincón, 2018), en el mismo se aporta una concepción del desarrollo de la compresión de los objetos de la Geometría Plana, en la que se devela la lógica didáctica entre los procesos de orientación motivacional-pedagógica de la comprensión de los objetos de la Geometría Plana; el manejo didáctico-situacional de los objetos de la Geometría Plana, y la valoración profesionalizante de la comprensión de los objetos de la geometría plana en el desempeño.

A continuación, se expresan las ideas que se derivan de la modelación realizada para el tratamiento didáctico de la comprensión de los objetos de la Geometría Plana: 
- Atender la enseñanza de la comprensión de los objetos de la Geometría Plana centrada en los procesos cognitivos, comunicativos y socioculturales en los que el lenguaje matemático es una herramienta útil para la comunicación, el cual se enseña y se utiliza en diferentes contextos, enfatizándose en su uso en los ambientes propios del estudiante, donde los procesos de significación y representación juegan un papel fundamental.

- Favorecer el proceso de comprensión de los objetos de la Geometría Plana como componente funcional del contenido geométrico, a través de situaciones didácticomatemáticas que generen conflictos cognitivos y permitan analizar los objetos de la Geometría Plana, significar en ellos lo esencial, establecer relaciones y operar con estos en la medida que se utilizan diferentes registros de representación y se argumenta matemáticamente teniendo en cuenta el contexto donde se utilicen.

- Favorecer la utilización de métodos y estrategias que hagan posible el desarrollo de motivos e intereses con énfasis en aquellos relacionados con la actividad profesional de los futuros egresados y un clima que favorezca las interacciones y la comunicación.

- Propiciar el desarrollo de una cultura valorativa de la comprensión en el desempeño mediante diferentes formas de evaluación, con énfasis en la autovaloración, covaloración utilizando criterios.

En la propuesta, a partir de los criterios valorativos generales para la idoneidad afectivainteraccional-mediacional, se definen criterios particulares para evaluar el desarrollo de la comprensión de los objetos de la Geometría Plana de los estudiantes de la carrera de educación, Mención Matemática (Rincón, Valenzuela, 2017).

Modelo orientado a la gestión didáctica de las demostraciones matemáticas (Núñez, 2018), en este se aporta una novedosa concepción de la gestión didáctica de las demostraciones matemáticas que tiene en cuenta los factores socioculturales que inciden en el desarrollo de las demostraciones matemáticas y en la que se devela la lógica didáctica entre los procesos de orientación-autoformativa del contenido didáctico-matemático para el tratamiento de las demostraciones matemáticas, proyección didáctico-situacional de la enseñanza-aprendizaje de las demostraciones matemáticas y el de valoración reflexiva- contextualizada del desempeño donde se precisan los criterios para evaluar el desempeño del profesor en la gestión didáctica de las demostraciones matemáticas.

El diseño de situaciones didáctico-matemáticas, se concibe como el núcleo de la gestión didáctica de las demostraciones matemáticas, y son expresión de un proceso de reconstrucción de las concepciones y relaciones didáctico-matemáticas en el trabajo con el contenido específico de las asignaturas (Núñez, Montes de Oca 2017). 
De esta forma las situaciones didáctico-matemáticas constituyen un recurso metodológico importante para la formación didáctico-matemática de los docentes, ya que estas tienen una intencionalidad didáctica en correspondencia con el contenido didáctico-matemático específico; que, en este caso, está relacionado con la enseñanza-aprendizaje de las demostraciones matemáticas y con la práctica reflexiva como elemento consustancial de esta formación.

En el contexto de la investigación, las situaciones didáctico-matemáticas que se diseñaron, estuvieron orientadas a sensibilizar sobre el significado de las demostraciones y su importancia, identificar los conocimientos necesarios y recursos didácticos que garantizan la comprensión de las demostraciones matemáticas, construir y reconstruir estrategias didácticas para el trabajo con las demostraciones matemáticas, utilizar preguntas guías que orienten el trabajo con diferentes métodos de demostración, utilizar los asistentes matemáticos para la exploración, formulación de conjeturas y la visualización, y propiciar la contrastación de opiniones a través del diálogo para reflexionar sobre los errores en el proceso de demostración (intercambiar, preguntar, discutir, socializar).

En la propuesta a partir de los criterios valorativos generales para la idoneidad epistémica-ecológica-cognitiva se definen criterios específicos para valorar el desempeño del profesor en la gestión didáctica de las demostraciones matemáticas (Núñez, Montes de Oca, 2016).

Modelo didáctico de producción de problemas durante el proceso de enseñanza aprendizaje del Álgebra Lineal (Pérez, 2018), constituye un sistema estructurado a través de los procesos de cimentación del contenido, de variación de problemas de Álgebra Lineal y de distinción de los problemas en producción. La sinergia entre los componentes del modelo se manifiesta en la significatividad lógica funcional, como nueva cualidad del sistema que expresa la formación del educando en el proceso, donde se considera el aprovechamiento de la potencialidad de los modelos algebraicos para la obtención de problemas significativos en la variación de problemas del Álgebra Lineal, como la solución a la contradicción relativa al conocimiento de la base de contenido; por medio del tratamiento didáctico, de manera progresiva, de la célula generadora del contenido del proceso de enseñanza aprendizaje y de la obtención de problemas (con su oportuna distinción) por los estudiantes. 
De la modelación realizada se infiere que es necesario tomar en cuenta la base de contenido propia del estudiante para su realización, y debe estar dirigido a la obtención de problemas significativos para un estudiante que se prepara como futuro maestro de Matemática. Aquí se muestra que se puede aprovechar la funcionalidad de los objetos del Álgebra Lineal, específicamente de los modelos algebraicos, para realizar las transformaciones en los problemas y para caracterizar al problema transformado.

Modelo de formación de la competencia docente promoción de la valoración positiva de la matemática (Báez, 2018), el mismo se estructura a través de tres subsistemas: procesos formativos aprehensivos de la problemática afectiva matemática; actuacional-áulico para la educación afectiva matemática; actuacional-contextual para la educación afectiva matemática y formativo de la afectividad matemática, de cuyas relaciones resulta la idoneidad pedagógica del estudiante para maestro como promotor de valoraciones positivas de la Matemática, como cualidad para identificar e interpretar los problemas afectivos matemáticos de sus estudiantes y propios, así como las características del contexto, para diseñar, implementar y valorar soluciones a estos problemas tanto desde el aula como desde la familia y escuela, para que en ese accionar ir fomentando una visión y una competencia percibida positiva de la matemática.

Como resultado de la investigación se ofrecen las dimensiones esenciales de la competencia docente promoción de la valoración positiva de la matemática, que se expresan a continuación:

- Comprensión de los problemas afectivos matemáticos: Relacionada con la identificación e interpretación de los problemas asociados al dominio afectivo de la matemática de los alumnos y del contexto familiar y escolar. Implica la formación en los ámbitos del dominio afectivo matemático, su problemática y el diagnóstico de la misma.

- Actuación didáctica en la dimensión afectiva: Relacionada con el diseño, implementación y valoración de la solución de los problemas de los alumnos identificados en el dominio afectivo en el espacio áulico. Implica su formación en los ámbitos del dominio afectivo matemático, su problemática y el tratamiento didáctico y psicológico para la solución de este tipo de problemas.

- Intervención en el contexto para la educación afectiva: Relacionada con el diseño, implementación y valoración de la solución de los problemas de los alumnos y del contexto familiar y escolar, fuera del espacio áulico. Implica la formación en los ámbitos del dominio afectivo matemático, su problemática y el tratamiento didáctico. psicológico y sociológico para la solución de este tipo de problemas. 
- Alfabetización afectiva matemática: Relacionada con la conceptualización del dominio afectivo de la matemática y su enseñanza; y la identificación, regulación y utilización que hace el docente de sus propias creencias, actitudes y emociones en este ámbito. Implica la formación en los ámbitos del dominio afectivo matemático, su problemática, la metacognición, la autorreflexión, la autorregulación y la reflexión sobre la práctica.

En la propuesta, a partir de los criterios valorativos generales para la idoneidad afectivainteraccional-mediacional, se definen criterios específicos para valorar el desempeño del profesor en la promoción de la valoración positiva de la Matemática.

Modelo didáctico de formación de la competencia dominio de conceptos básicos del Álgebra Lineal en la licenciatura en educación mención Matemática (Almonte, 2018), incluye los subsistemas representación cognitiva-actitudinal de conceptos básicos e instrumentación didáctico-técnica del dominio de los conceptos algebraicos, que constituyen respectivamente la representación teórico e instrumental de la formación de la competencia. Modela la aprehensión de los saberes algebraicos al ofrecer herramientas de aprendizaje a los estudiantes que facilitan la asimilación de lo esencial y la aplicación de los conceptos, de forma que se resalta lo afectivo. Se argumenta la elaboración de situaciones de aprendizaje por parte del docente para que los estudiantes construyan tareas específicas relacionadas con su aprendizaje y el vínculo profesional, de manera protagónica y responsable. Se connota que, de las relaciones de coordinación establecidas entre los subsistemas y componentes del modelo, se sistematiza la problematización de los conceptos algebraicos.

En el proceso investigativo se delimitaron como unidades que componen esta competencia, las siguientes:

- La comprensión del concepto escolar: Relacionada con la identificación de los conceptos algebraicos del Álgebra Lineal que se estudian en la Educación Media, las características y propiedades que los forman, mediante el empleo de medios de aprendizaje virtuales, el análisis de los objetos algebraicos en sus diferentes representaciones de forma crítica y creativa, la identificación de las características necesarias y suficientes que los definen, a través de herramientas tecnológicas.

- La generalización del concepto: Relacionada con la construcción de ejemplos y contra ejemplos, casos límites y especiales, a determinación de relaciones existentes entre objetos algebraicos, la utilización del contenido y extensión del concepto, a comparación crítica de varias definiciones del concepto, e establecimiento de relaciones entre concepto superior y concepto subordinado y la clasificación de los objetos algebraicos estudiados a partir de sus características esenciales, con el uso de las tecnologías de la información y las comunicaciones. 
- Aplicación del concepto: Relacionada con la elección con rigor y seguridad de las propiedades adecuadas que facilitan la toma de decisiones en la selección de la vía de solución, y con el empleo del concepto en diversas situaciones y la solución de problemas con creatividad y responsabilidad

El modelo toma en consideración las implicaciones de las representaciones semióticas en la realización de actividades matemáticas, su influencia en el mejoramiento del desempeño de los estudiantes en el dominio de los conceptos en su aprendizaje matemático y para su ejercicio profesional. Se tiene en cuenta la mediación de la tecnología para facilitar la dirección del proceso de apropiación de los conceptos (Almonte; García, 2016).

En la propuesta a partir de los criterios valorativos generales para la idoneidad epistémica-ecológica-cognitiva se definen criterios específicos para valorar el desempeño del en el dominio de conceptos básicos del Álgebra Lineal.

\section{Modelo didáctico de la formación matemática didáctica del docente en Educación}

Primaria (Lachapell, 2018), constituye un sistema que se estructura en tres subsistemas: procesos de formación en los contenidos matemáticos, procesos de formación en los contenidos didácticos de la matemática del nivel primario y procesos de análisis integrador de la práctica matemático-didáctica, de cuya sinergia resulta la interactividad cognitiva matemático didáctica, como nueva cualidad del sistema que posibilita que el docente en formación se ubique en las acciones que debe realizar para lograr la consecución de los objetivos de aprendizaje y apropiarse del contenido matemático y didáctico en unidad dialéctica, mediante la aplicación de un pensamiento y razonamiento matemático junto con un dominio de las operaciones matemáticas simbólicas y formales para desarrollar nuevas estrategias y para enfrentarse a resolver situaciones nuevas.

En el contexto de la investigación las situaciones didáctico-matemáticas constituyen la célula del proceso, a través de la cual se va generando la formación. A partir de estas, la integración del contenido matemático y el contenido didáctico se desarrolla desde la interpretación particular y contextual que los futuros docentes hacen de la situación a partir de sus creencias, intuiciones, experiencias, vivencias, saberes, en una estrecha relación con el grupo que posibilita sucesivas reinterpretaciones en dicha integración.

De esa forma las situaciones didáctico-matemáticas permiten la elaboración del conocimiento, la planificación de sus recursos funcionales y estratégicos y, analizar a qué obedece la selección estratégica realizada mediante la ejecución de las situaciones que ellos 
deben resolver. A su vez, deben permitir la reflexión didáctica sobre el contenido matemático, el estudio de las investigaciones didácticas sobre errores y dificultades de aprendizaje; sobre métodos y recursos de enseñanza y su realización práctica. Así como, la confrontación y validación de las propias creencias y concepciones frente a los resultados producidos por la investigación didáctica.

En la propuesta, a partir de los criterios valorativos generales para la idoneidad didáctica, se definen criterios específicos para valorar la formación matemática didáctica del docente de Educación Primaria.

\section{Reflexiones finales}

La novedad de los resultados se expresa en develar la concepción de la formación didáctico-matemática, integrando la perspectiva histórico - cultural del desarrollo humano, con los enfoques ontosemiótico, de los cambios de registros de representación semiótica, comunicativo y contextualizado de la enseñanza-aprendizaje de la Matemática.

De las tesis en opción al grado de Doctor en Ciencias Pedagógicas (Rincón, 2018; Núñez, 2018; Pérez, 2018; Báez, 2018; Almonte, 2018; Lachapell, 2018) resultantes de la investigación, se derivaron estrategias que contribuyeron a mejorar el desempeño de estudiantes y docentes en la gestión didáctica de objetos y procesos matemáticos, en la promoción de una valoración positiva de la Matemática, en la comprensión y resolución de problemas de Geometría Plana y de Álgebra Lineal y en la expresión al comunicarse en Matemática en diversos contextos y escenarios educativos.

Los resultados de la investigación fueron sometidos a la valoración de la Comisión de Ciencias Sociales de la Academia de Ciencias de Cuba, y fue seleccionada Premio Nacional a los resultados de la investigación científica del año 2019.

Por la importancia del tema, es preciso continuar profundizando en los constructos aportados y su necesaria articulación para lograr cada vez más la precisión que requiere su introducción en la práctica dentro de los programas de formación de docentes.

Los resultados responden a la labor realizada por un grupo de investigadores del Proyecto Perfeccionamiento de la enseñanza de la Matemática del Programa Nacional Problemas Actuales del Sistema Educativo Cubano, perspectivas de desarrollo. 


\section{Agradecimientos}

Gracias a Isabel Yordi González por compartir conmigo el liderazgo de esta investigación, a Olga Lidia Pérez González por la excelente coordinación del proyecto, a Delia Sarduy Nápoles por su incondicional ayuda y a todos los que participaron por haber hecho posible esta publicación.

\section{Referencias}

Almonte, R.M. (2018). Formación de la competencia dominio de conceptos básicos del álgebra lineal en la Licenciatura en Educación mención Matemática. Camagüey: (Tesis doctoral). Universidad de Camagüey Ignacio Agramonte Loynaz.

Almonte, R.M. (2016). Descripción de la competencia dominio de conceptos básicos en estudiantes de educación matemática. Revista IPLAC. No 5. www.revista.iplac.rimed.cu

Almonte, R.M. y García, J. (2016). Panorama de la integración de la tecnología de la información y la comunicación (TIC) en el proceso enseñan aprendizaje en la República Dominicana. Revista IPLAC, No.1.

Báez, I. (2018). Formación de la competencia docente promoción de la valoración positiva de la matemática en la formación inicial de maestros del nivel primario. Camagüey: (Tesis doctoral). Universidad de Camagüey Ignacio Agramonte Loynaz.

Báez, I., Legañoa, M., y García, J. (2017). Las competencias de los maestros para resolver los problemas afectivos matemáticos de los estudiantes. Pedagogía Profesional, 15(2)

Báez, I., Legañoa, M., y García, J. (2018). Formación inicial de maestros en la competencia promoción de la valoración positiva de la matemática: un estudio de casos. Transformación, 14 (2): 262- $271 .$.

Báez, I. (2016). Nuevo enfoque de la formación inicial pedagógica matemática de maestros de educación primaria. Revista IPLAC, No. 5.

Breda, A., Font, V. y Pino-Fan, L. (2018). Criterios valorativos y normativos en la Didáctica de las Matemáticas: el caso del constructo idoneidad didáctica. BOLEMA, 60(1): 255 - 278.

Byas, R y Blanco, R. (2017). Didáctica de la Matemática en la formación docente. Santo Domingo: Editora universitaria.

Camarena, P. (2009) La Matemática en el contexto de la ciencia. México. Revista Innovación educativa: Las Matemáticas y la educación. 9 (46): 15-23.

Duval, R. (1999). Registros de representación semiótica y funcionamiento cognitivo del pensamiento. Investigaciones en Matemática Educativa II, 173-201. México: Grupo Editorial Iberoamérica.

Duval, R. (2006). Un tema crucial en la educación matemática: La habilidad para cambiar el registro de representación. La Gaceta de la Real Sociedad Matemática Española, 9 (1): 143 -168 .

Font, V. y Godino, J. (2006). La noción de configuración epistémica como herramienta de análisis de textos matemáticos: su uso en la formación de docentes. Brasil: Educaçao Matemática Pesquisa. 8 (1): 67-98. 
Font, V. (2011). Competencias profesionales en la formación inicial de docentes de matemáticas de secundaria. San Cristóbal de La Laguna: Revista Iberoamericana de educación matemática. Unión. (26): 9-25.

Godino, J., Bencomo, D., Font, V., y Wilhelmi, M. (2007). Análisis y valoración de la idoneidad didáctica de procesos de estudio de las matemáticas. Paradigma, 27 (2): 221-252.

Godino, J. (2009). Categorías de Análisis de los conocimientos del Docente de Matemáticas. España: Revista Iberoamericana de educación matemática. Unión, (20): 13-31.

Godino, J. (2013). Indicadores de idoneidad didáctica de procesos de enseñanza y aprendizaje de las matemáticas. Cuadernos de Investigación y Formación en Educación Matemática, 8(11): 111-132.

González, M. (2010). Estrategia didáctica sustentada en un modelo matemático-comunicativo para favorecer la argumentación en matemática. Camagüey: (tesis doctoral). Universidad de Camagüey Ignacio Agramonte Loynaz.

Lachapell, Y.A. (2018). La formación matemática didáctica del docente en educación primaria de la República Dominicana. Camagüey: (Tesis doctoral). Universidad de Camagüey Ignacio Agramonte Loynaz. Cuba.

Lachapell, Y. (2017). La formación didáctico matemática del docente de la República Dominicana. Transformación, 13 (3): 364-373.

Lachapell, Y., Mola, C. y Quintero, G. (2016). Tareas de aprendizaje para la sistematización formativa del conocimiento didáctico matemático en la educación inicial del maestro dominicano. Revista IPLAC. No. 3.

Legañoa, M., Baez, I. y García, J. (2017). Las actitudes hacia la matemática: preparación de los maestros para considerarlas. Transformación, 13 (1): 57-66.

Machado, E. y Montes de Oca, N. (2016). Formación profesional y desempeños idóneos del docente universitario. Humanidades Médicas. 16 (1): 1-20

Montes de Oca, N. y Machado, E. (2014). Formación y desarrollo de competencias en la educación superior cubana. Humanidades Médicas. 14 (1): 1-20.

Moura, M. (2011). Educar con las matemáticas: saber específico y saber pedagógico. Educación y Pedagogía. 23(59): 47-57.

Núñez, G. (2018). La formación didáctico-matemática de los docentes desde el ejercicio de la profesión orientada a la gestión didáctica de las demostraciones matemáticas. Camagüey: (Tesis doctoral). Universidad de Camagüey Ignacio Agramonte Loynaz.

Núñez, G. y Montes de Oca, N. (2016). Criterios para evaluar el desempeño docente en la gestión didáctica de las demostraciones matemáticas. Revista IPLAC. No 3.

Núñez, G. y Montes de Oca, N. (2017). Las demostraciones matemáticas y su gestión didáctica. Revista IPLAC. No. 5.

Pérez, A. (2017). Concepción y diseño de tareas asociadas a las aplicaciones del contenido de Álgebra superior en carreras de Educación Matemática. Revista IPLAC. No. 4. 
Pérez, A. (2018). Producción de problemas de álgebra lineal para futuros maestros de matemática. Camagüey: (Tesis doctoral). Universidad de Camagüey Ignacio Agramonte Loynaz.

Pérez, A., Yordi, I. y García, J. (2018). Algebra for the initial training of Mathematics teachers at Autonoma University of Santo Domingo. Journal Magazine of Education and Social Policy (JESP). 1: 97- 99.

Pino-Fan, L. Castro, W. Godino, J. y Font, V. (2013). Idoneidad epistémica del significado de la derivada en el currículo de bachillerato. Paradigma. 34 (2): 123-150.

Rincón, E. (2018). El desarrollo de la comprensión de los objetos de la geometría plana en los estudiantes de la carrera de educación, mención Matemática. Camagüey: (Tesis doctoral). Universidad de Camagüey Ignacio Agramonte Loynaz.

Rincón, E., Montes de Oca, N. y Mola, C. (2017). Estrategia para la comprensión de los objetos de la Geometría Plana en la carrera de Educación mención Matemática. Didasc@lia: Didáctica y Educación. VIII (4): 179-189.

Rincón, E. y Valenzuela Y. (2017). Niveles y criterios de logro para evaluar el desarrollo de la comprensión de los objetos de la Geometría.

Rincón, E., Montes de Oca, N. y Mola, C. (2018) Estrategia para la comprensión de los objetos geométricos, en la carrera de Educación, mención Matemática. Didasc@lia: Didáctica y Educación. VIII (4): 179-197.

Roméu, A. (2011). El enfoque cognitivo, comunicativo y sociocultural de la enseñanza de la lengua: periodización y aportes. Habana: (tesis doctoral) UCP "Enrique José Varona".

Sobrado, E., Sarduy, D. y Montes de Oca, N (2016). Evaluación de la expresión del profesor en formación al comunicarse en matemática. Transformación. 12 (1). 101-111.

Sobrado, E., Sarduy, D. y Espíndola, A. (2018). Estrategia didáctica para mejorar la calidad de la comunicación en matemática. Transformación. 14 (2): 272-285.

Valenzuela, Y., Montes de Oca N. y Pérez, O. (2019). Entrenamiento para la formación didáctico-matemática de los docentes del nivel secundario Didasc@lia: Didáctica y Educación. X(2). 165-179

Vigotsky, L. S. (1987). Historia del desarrollo de las funciones psíquicas superiores. La Habana: Editorial Científico Técnica. 